Pacific Journal of Mathematic 


\title{
ON THE LATTICE OF ALL CLOSED SUBSPACES OF A HERMITIAN SPACE
}

\author{
HANS A. KELLER
}

The purpose of the paper is to prove the following

Theorem: Let $E$ be a vector space over a field $K$ with char $K \neq 2$, and let $\phi$ be a nondegenerate hermitian form on $E$. Then the lattice of all orthogonally closed subspaces of $(E, \phi)$ is modular if and only if $E$ is finite dimensional.

Introduction. It is well known that the lattice of all orthogonally (=topologically) closed subspaces of a Hilbert space $H$ is modular only if $H$ has finite dimension (see Birkhoff-Von Neumann [1]). We shall prove here that this is true generally for vector spaces $E$ over commutative fields $K$ with char $K \neq 2$, supplied with nondegenerate hermitian forms $\phi$ : The lattice of all orthogonally closed subspaces of $(E, \phi)$ is modular if and only if $E$ is finite dimensional. Nonmodularity in the infinite dimensional case is due to the fact that then there are always two closed subspaces with nonclosed sum. In a Hilbert space one can exhibit such pairs of subspaces in a constructive way (see [3]); our general case is much more involved, and their existence will follow from an indirect proof.

1. Denotations. Let $E$ be a (left-) vector space over a commutative field $K$, and $\phi: E \times E \rightarrow K$ a hermitian form with respect to an automorphism $\alpha \mapsto \bar{\alpha}$ of period 2 of $K$. We always assume that char $K \neq 2$. We usually write $(x, y)$ instead of $\phi(x, y)$, and we write $x \perp y$ if $(x, y)=0, x, y \in E$. Let $F$ be a subspace of $(E, \phi)$. The orthogonal space of $F$ is $F^{\perp}=\{x \in E: x \perp y$ for all $y \in F\}$, and the radical of $F$ is $\operatorname{rad} F=F \cap F^{\perp} . \quad F$ is called semisimple if $\operatorname{rad} F=0$. In particular, $E$ is semisimple if $E^{\perp}=0$, i.e., if $\phi$ is nondegenerate. A subspace $F$ is called orthogonally closed if $F=$ $F^{\perp \perp}\left(=\left(F^{\perp}\right)^{\perp}\right)$. All bases of vector spaces are algebraic. $F$ is termed euclidean if it is semisimple and admits an orthogonal basis. Semisimple subspaces of countable dimension are always euclidean (see [2]). Every $x \in E$ induces a linear form $\phi_{x}$ on $F$, given by $\phi_{x}(z)=$ $\phi(z, x), z \in F$. We let $F^{*}$ denote the antispace of the dual space of $F$, i.e., the $K$-space of all linear forms $f: F \rightarrow K$, where $(f+g)(z)=$ $f(z)+g(z)$ and $(\alpha f)(z)=\bar{\alpha} \cdot f(z), f, g \in F^{*}, \alpha \in K$. If $F^{\perp}=0$ then $E$ can be considered as a subspace of $F^{*}$, identifying $x \in E$ with $\phi_{x}$.

If $E=\bigoplus_{i \in I} E_{i}$, and $E_{i} \perp E_{j}$ for $i \neq j$, we write $E=\bigoplus_{i \in I}^{{ }^{L}} E_{i}$.

2. The lattice $\mathscr{L}(E, \dot{\phi})$. Let $(E, \phi)$ be a semisimple hermitian 
space over $K$. The orthogonally closed subspaces of $(E, \phi)$ form a lattice $\mathscr{L}=\mathscr{L}(E, \dot{\phi})$ under the operations $F \wedge G=F \cap G$ and $F \vee G=$ $(F+G)^{\perp \perp}$. This lattice is modular iff for all $F, G \in \mathscr{L}$ we have $F \vee G=F+G$ (see [4], Theorem 33.4). Thus modularity of $\mathscr{L}(E, \phi)$ is equivalent to the following property of $(E, \phi)$ :

(A) The sum of two orthogonally closed subspaces is always closed .

If $\operatorname{dim} E<\infty$ then (A) holds trivially. We now prove the converse.

3. Nonmodularity of $\mathscr{L}(E, \phi)$ in case of infinite dimension. We start with two technical lemmas. Their importance for our problem will become evident later (cf. the proof of Lemma 3 below).

Lemma 1. Let $(E, \dot{\phi})$ be semisimple. Let $F$ be a subspace with $\operatorname{dim} F=\$_{0}$ such that for all subspaces $U, V \subset F$ we have: If $U+V=F$ then $U^{\perp \perp}+V^{\perp \perp}=E$. Then $F=E$.

Proof. Taking $U=V=F$ we get $F^{\perp \perp}=E$ and $F^{\perp}=0$. Therefore $E$ may be considered as a subspace of $F^{*}$. Let $F=\bigoplus_{s \in S}^{\perp} F_{s}$ be an orthogonal decomposition of $F$ into finite dimensional subspaces and let $y \in F^{*} . y$ is determined by the restrictions $\left.y\right|_{F_{8}}$. Every $F_{s}$ is semisimple since $F^{\perp}=0$, thus $\left.y\right|_{F_{s}}$ is induced by a unique $y_{s} \in F_{s}$. This allows us to represent $y$ as a formal sum $y=$ $\sum_{s \in S} y_{s}$, and we call the $y_{s}$ 's the components of $y$ with respect to the decomposition $F=\bigoplus_{s} F_{s}$. In particular every $x \in E$ has the form $x=\sum_{s} x_{s}$.

Now suppose that $E \neq F$.

(1) We first show that then $E=F^{*}$. Let $x \in E$ with $x \notin F$. One readily constructs a decomposition $F=\bigoplus_{S}^{1} F_{s}$ such that $\operatorname{dim} F_{s}=2$ and $x_{s} \neq 0$ for all $s \in S$ (choose an orthogonal basis $\left\{e_{i}: i \in I\right\}$ of $F$ and observe that $\left.\operatorname{card}\left\{i \in I:\left(e_{i}, x\right) \neq 0\right\}=\aleph_{0}=\operatorname{card} I\right)$. Now let $y \in F^{*}$. We write $y=\sum_{s} y_{s}$, where $y_{s} \in F_{s}$, and suppose first that $\left\{x_{s}, y_{s}\right\}$ is linearly independent for all $s$. Let $U$ and $V$ be the subspaces spanned by $\left\{y_{s}: s \in S\right\}$ and $\left\{x_{s}-y_{s}: s \in S\right\}$ respectively. We have $U+V=F$, thus $U^{\perp \perp}+V^{\perp \perp}=E$. Write $x=u+v$, where $u=\sum_{s} u_{s} \in U^{\perp \perp}$ and $v=\sum_{s} v_{s} \in V^{\perp \perp}\left(u_{s}, v_{s} \in F_{s}\right)$. Pick $a_{s} \in F_{s}$ with $a_{s} \neq 0$ and $a_{s} \perp y_{s}$. Then $a_{s} \in U^{\perp}$, hence $0=\left(a_{s}, u\right)=\left(a_{s}, u_{s}\right)$. Since $\operatorname{dim} F_{s}=2$ it follows that $u_{s}=\lambda_{s} y_{s}$ for some $\lambda_{s} \in K$. In the same way we get $v_{s}=\mu_{s}\left(x_{s}-y_{s}\right), \mu_{s} \in K$. Since $u_{s}+v_{s}=x_{s}$ we have $\lambda_{s}=\mu_{s}=1$. Thus $y_{s}=u_{s}$ for all $s$, hence $y=u$ and in particular $y \in E$ in this case. Next we consider $y=\sum_{s} y_{s}$ in $F^{*}$ with $y_{s} \neq 0$ for all $s$. For every $s$ choose $z_{s} \in F_{s}$ such that $\left\{x_{s}, z_{s}\right\}$ and $\left\{z_{s}, y_{s}\right\}$ are both linearly independent. Applying the above reasoning to $x$ and $z=\sum_{s} z_{s} \in F^{*}$ as well as to $z$ and $y$ we get first $z \in E$ and 
then $y \in E$. The $y$ 's in $F^{*}$ with all components $\neq 0$ generate $F^{*}$. Since all these $y$ 's are in $E$ we have $E=F^{*}$.

(2) Suppose $F=\bigoplus_{s}^{+} F_{s}$, where $\operatorname{dim} F_{s}<\aleph_{0}$ for all $s$. Let $x=\sum_{s} x_{s}, y=\sum_{s} y_{s}$ be in $E, x_{s}, y_{s} \in F_{s}$. We claim: if $\left(x_{s}, y_{s}\right)=0$ for all $s$, then $(x, y)=0$. To prove this let $U$ and $W$ be the subspaces generated by $\left\{x_{s}: s \in S\right\}$ and $\left\{y_{s}: s \in S\right\}$ respectively. We have $U \perp W$, hence $U^{\perp \perp} \perp W^{\perp \perp}$. Therefore it is enough to show that $x \in U^{\perp \perp}$ and $y \in W^{\perp \perp}$. Choose linear complements $V_{s}$ of $\left(x_{s}\right)$ in $F_{s}$, $F_{s}=V_{s} \oplus\left(x_{s}\right)$, and put $V=\bigoplus_{S}^{\perp} V_{s}$. Then $U+V=F$, hence $U^{\perp 1}+V^{\perp \perp}=E$. Write $x=u+v$, where $u=\sum_{s} u_{s} \in U^{\perp \perp}, v=$ $\sum_{s} v_{s} \in V^{\perp \perp}$. For every $z_{s} \in F_{s}$ with $z_{s} \perp x_{s}$ we have $z_{s} \in U^{\perp}$ and so $0=\left(z_{s}, u\right)=\left(z_{s}, u_{s}\right)$. This gives $u_{s} \in\left(x_{s}\right)^{\perp \perp}=\left(x_{s}\right)$. In the same way we get $v_{s} \in V_{s}^{\perp \perp}=V_{s}$. Since $u_{s}+v_{s}=x_{s}$ it follows that $v_{s}=0$ and $u_{s}=x_{s}$. Thus $x=u \in U^{\perp \perp}$. In the same way we see that $y \in W^{\perp \perp}$.

(3) Let $\left\{e_{i}: i \in I\right\}$ be an orthogonal basis of $F$. According to $F=\bigoplus_{I}^{\perp}\left(e_{i}\right)$ every $x \in E=F^{*}$ can be written in the form $x=\sum_{i} \xi_{i} \cdot e_{i}$ with $\xi_{i}=\left(\overline{\left.e_{i}, x\right)}\left(e_{i}, e_{i}\right)^{-1}\right.$. For $T \subset I$ we put $x_{T}=\sum_{i} \xi_{i}^{\prime} e_{i}$, where $\xi_{i}^{\prime}=\xi_{i}$ for $i \in T$ and $\xi_{i}^{\prime}=0$ for $i \notin T$. We consider $a=\sum_{i} \alpha_{i} e_{i}$ and $b=$ $\sum_{i} \beta_{i} e_{i}$, where $\alpha_{i}=\left(e_{i}, e_{i}\right)^{-1}$ and $\beta_{i}=1$ for all $i . a, b \in E$ by (1). Let $I=S \cup T$ be a partitioning with card $S=\operatorname{card} T$. We show that

$$
\left(a_{S}, b_{S}\right)=\left(a_{T}, b_{T}\right) \text {. }
$$

We observe that $\left(a_{S}, b_{T}\right)=\left(a_{T}, b_{S}\right)=0$ by (2). Thus it suffices to show that $a=a_{S}+a_{T}$ and $c=b_{S}-b_{T}$ are orthogonal. Let $\sigma: S \rightarrow T$ be a bijection. For $s \in S$ put $F_{s}=K\left(e_{s}, e_{\sigma s}\right)$. Then $F=\bigoplus_{S}^{\frac{1}{s}} F_{s}$. The corresponding components of $a$ and $c$ are $a_{s}=\left(e_{s}, e_{s}\right)^{-1} \cdot e_{s}+\left(e_{s s}, e_{\sigma s}\right)^{-1}$. $e_{\sigma s}$ and $c_{s}=e_{s}-e_{\sigma s}$. We find $\left(a_{s}, c_{s}\right)=0$, and by (2) this implies $(a, c)=0$, as claimed.

We now choose $t \in T$ and put $S^{\prime}=S \cup\{t\}$ and $T^{\prime}=T-\{t\}$. We have card $S^{\prime}=\operatorname{card} T^{\prime}$, hence $\left(a_{S^{\prime}}, b_{S^{\prime}}\right)=\left(a_{T^{\prime}}, b_{T^{\prime}}\right)$. On the other hand, from the relations $a_{S^{\prime}}=a_{S}+\left(e_{t}, e_{t}\right)^{-1} \cdot e_{t}, a_{T^{\prime}}=a_{T}-\left(e_{t}, e_{t}\right)^{-1} \cdot e_{t}$ and $b_{S^{\prime}}=b_{S}+e_{t}, b_{T^{\prime}}=b_{T}-e_{t}$ we get

$$
\left(a_{S^{\prime}}, b_{S^{\prime}}\right)=\left(a_{S}, b_{S}\right)+1, \quad\left(a_{T^{\prime}}, b_{T^{\prime}}\right)=\left(a_{T}, b_{T}\right)-1 \text {. }
$$

It follows that $+1=-1$, a contradiction since char $K \neq 2$. This completes the proof.

We can easily generalize the statement of Lemma 1 .

Lemma 2. Let $(E, \phi)$ be semisimple. Let $F$ be a euclidean subspace such that whenever $U+V=F$ it follows that $U^{\perp \perp}+V^{\perp \perp}=E$. Then $F=E$.

Proof. Since $F^{\perp \perp}=E$ we may suppose that $\operatorname{dim} F \geqq \boldsymbol{\aleph}_{0}$. Let 
$\left\{e_{i}: i \in I\right\}$ be an orthogonal basis of $F$. Suppose that there exists a $x \in E$ with $x \notin F$. Then there exists a subset $L \subset I$ with $\operatorname{card} L=\aleph_{0}$ and 'such that $\left(e_{i}, x\right) \neq 0$ for all $i \in L$. Put $Q=K\left(e_{i}\right)_{i \in L}$ and $R=$ $K\left(e_{i}\right)_{i \in I-L}$; then $F=Q \bigoplus^{\perp} R$ and so $E=Q^{\perp \perp} \bigoplus^{\perp} R^{\perp \perp}$. Write $x=$ $q+r$ where $q \in Q^{\perp \perp}, r \in R^{\perp \perp}$. One easily verifies that the hypotheses of "Lemma 1 are satisfied for $\left(Q^{\perp \perp},\left.\phi\right|_{Q \perp \perp}\right)$ and $Q$ (in lieu of $(E, \phi)$ and $F)$. Hence $Q=Q^{\perp \perp}$ and in particular $q \in Q$. But this is a contradiction since $\left(e_{i}, q\right)=\left(e_{i}, x\right) \neq 0$ for all $i \in L$.

We now pass to study spaces $(E, \phi)$ with property (A).

LeMma 3. Suppose that the semisimple space $(E, \phi)$ has property (A). Then for every euclidean subspace $F$ we have $F^{1 \perp}=$ $F \bigoplus^{\perp} \operatorname{rad} F^{\perp}$.

Proof. We have $\operatorname{rad} F^{\perp \perp}=\operatorname{rad} F^{\perp}$, and $F \cap F^{\perp}=0$. Hence there is a decomposition $F^{\perp \perp}=Q \bigoplus^{\perp} \operatorname{rad} F^{\perp}$ with $F \subset Q$. The space $Q$ with the induced form $\Psi=\left.\phi\right|_{Q}$ (restriction) is semisimple. We shall show that the hypotheses of Lemma 2 are satisfied for $(Q, \Psi)$ and $F$ (in place of $(E, \phi)$ and $F$ ); then it will follow that $F=Q$, proving our lemma. For $U \subset Q$ we let $U^{\circ}$ denote the orthogonal space of $U$ formed in $(Q, \Psi)$. Thus $U^{\circ}=\{x \in Q: x \perp y$ for all $y \in U\}=$ $U^{\perp} \cap Q$. Now let $U, V$ be subspaces of $F$ with $U+V=F$; we must show that $U^{00}+V^{00}=Q$. It is immediate that $U^{00} \oplus \operatorname{rad} F^{\perp} \supset U^{\perp \perp}$ and $V^{00} \oplus \operatorname{rad} F^{\perp} \supset V^{\perp \perp}$. By (A), $U^{\perp \perp}+V^{\perp \perp}$ is closed in $(E, \phi)$, thus $U^{\perp \perp}+V^{\perp \perp}=\left(U^{\perp \perp}+V^{\perp \perp}\right)^{\perp \perp}=(U+V)^{\perp \perp}=F^{\perp \perp}$. It follows that $\left(U^{00}+V^{00}\right) \oplus \operatorname{rad} F^{\perp} \supset U^{\perp \perp}+V^{\perp \perp}=F^{\perp \perp}$, hence $U^{00}+V^{00}=Q$, as claimed.

Let $(H, \Psi)$ be any hermitian, euclidean space over $K$. We denote by $H_{0}^{*}$ the set of all linear forms $f$ on $H$ with the property that $\operatorname{ker}(f)$, as a subspace of $(H, \Psi)$, admits an orthogonal basis. Let $\left\{h_{i}: i \in I\right\}$ be an othogonal basis of $H$, and let $f$ be any linear form on $H$. Put $J=\left\{i \in I: f\left(h_{i}\right) \neq 0\right\} . \quad f$ is induced by some $x \in H$ iff $J$ is finite. In this case, of course, $f \in H_{0}^{*}$. Suppose $J$ is infinite. Then $\operatorname{ker}(f)$ is semisimple and we have $f \in H_{0}^{*}$ iff card $J=\boldsymbol{\aleph}_{0}$ ([2], Satz 1). We now see that $f \in H_{0}^{*}$ if and only if there is a decomposition $H=Q \bigoplus^{\perp} R$ with $\operatorname{dim} R \leqq \aleph_{0}$ and $\left.f\right|_{Q}=0$. In such a decomposition $Q$ is always euclidean (cf. [2]). We also see that $H_{0}^{*}$ is a subspace of $H^{*}$.

LEMMA 4. Suppose $(E, \phi)$ is semisimple and has property (A). Let $F$ be a euclidean subspace. Then every $f \in F_{0}^{*}$ is induced by some $y \in E$.

Proof. If $f$ is not induced by a $x \in F$ then $G=\operatorname{ker}(f)$ is semi- 
simple and thus, by definition of $F_{0}^{*}$, euclidean; furthermore $\operatorname{dim} F / G=1$. We have $F^{\perp} \neq G^{\perp}$, for otherwise by Lemma 3 we would have

$$
G \oplus \operatorname{rad} F^{\perp}=G \oplus \operatorname{rad} G^{\perp}=G^{\perp \perp}=F^{\perp \perp}=F \oplus \operatorname{rad} F^{\perp},
$$

which is impossible. Hence there is a $y \in G^{\perp}$ with $y \notin F^{\perp}$, and it is clear that $f$ is induced by a suitable multiple $\lambda y(\lambda \in K)$.

We are ready to prove our main result.

THEOREM. Let $(E, \dot{\phi})$ be a semisimple hermitian space over a commutative field $K$ with char $K \neq 2$. The lattice of all orthogonally closed subspaces of $(E, \phi)$ is modular if and only if $E$ is finite dimensional.

Proof. One half of the statement is clear. Suppose $\mathscr{L}(E, \phi)$ is modular. Then (A) holds for $(E, \phi)$. Let $M=\left\{v_{i}: i \in I\right\}$ be a maximal set of pairwise orthogonal anisotropic vectors of $E$ ( $x \in E$ is anisotropic if $(x, x) \neq 0)$. The subspace $F$ spanned by the $v_{i}$ 's is euclidean. By the maximality of $M$ we have $\left.\phi\right|_{F \perp}=0$, hence $\operatorname{rad} F^{\perp}=F^{\perp}$. Thus $F^{\perp \perp}=F \oplus F^{\perp}$ by Lemma 3. Now suppose that $\operatorname{dim} E \geqq \aleph_{0}$. Then $\operatorname{dim} F \geqq \aleph_{0}$ since $(E, \phi)$ is semisimple. Hence there exists an element $f \in F_{0}^{*}$ which is not induced by a $x \in F$. By Lemma $4, f$ is induced by some $y \in E$. Clearly $y \notin F \oplus F^{\perp}$; since $F \oplus F^{\perp}=F^{\perp \perp}$ there exists $v \in F^{\perp}$ with $(v, y) \neq 0$. Put $G=$ $F \oplus(y) \oplus(v)$. One readily verifies that $G$ is semisimple. Since $f \in F_{0}^{*}$ there is a decomposition $F=Q \oplus^{\perp} R$ such that $\left.f\right|_{Q}=0$ and $\operatorname{dim} R=\aleph_{0}$; here $Q$ is euclidean. We have $y \in Q^{\perp}$ and so $G=$ $Q \bigoplus^{\perp}(R \oplus(y) \oplus(v))$ which shows that $G$ is euclidean. We define a linear form $g$ on $G$ by $\left.g\right|_{F}=f, g(y)=0, g(v)=(v, y)+1$. The above decomposition of $G$ shows that $g \in G_{0}^{*}$. Hence $g$ is induced by some $z \in E$. Since $\left.g\right|_{F}=f$ we have $z-y \in F^{\perp}$, i.e., $z=y+w$ with $w \in F^{\perp}$. Now $(v, y)+1=g(v)=(v, z)=(v, y)+(v, w)$, hence $(v, w)=1$. But this is a contradiction since $v, w \in F^{\perp}$ and $\phi$ vanishes on $F^{\perp}$. This completes the proof.

\section{REFERENCES}

1. G. Birkhoff and J. Neumann, The logic of quantum mechanics, Ann. of Math., 37 (1936), 823-843.

2. H. Gross, Der Euklidische Defekt bei quadratischen Räumen, Math. Ann., 180 (1969), 95-137.

3. P. R. Halmos, Introduction to Hilbert Spaces and the Theory of Spectral Multiplicity, Chelsea, New York, 1957. 
4. F. Maeda and S. Maeda, Theory of Symmetric Lattices, Springer Verlag, New York, 1970.

Received May 10, 1979.

Instituto de Matematica

Universidad Catolica de Chile

Casilla 114-D

Santiago, Chile 


\section{PACIFIC JOURNAL OF MATHEMATICS}

\section{EDITORS}

DONALD BABBITT (Managing Editor)

University of Galifornia

Los Angeles, California 90024

Hugo RossI

University of Utah

Salt Lake City, UT 84112

C. C. MoOre AND ANDrew OGG

University of California

Berkeley, CA 94720

\section{J. DugundjI}

Department of Mathematics University of Southern California

Los Angeles, California 90007

R. Finn and J. Milgram

Stanford University

Stanford, California 94305

ASSOCIATE EDITORS

E. F. Beckenbachi

B. H. NeumanN

F. WOLF

K. YOSHIDA

\section{SUPPORTING INSTITUTIONS}

UNIVERSITY OF BRITISH COLUMBIA

CALIFORNIA INSTITUTE OF TECHNOLOGY

UNIVERSITY OF CALIFORNIA

MONTANA STATE UNIVERSITY

UNIVERSITY OF NEVADA, RENO

NEW MEXICO STATE UNIVERSITY

OREGON STATE UNIVERSITY

UNIVERSITY OF OREGON
UNIVERSITY OF SOUTHERN CALIFONIA

STANFORD UNIVERSITY

UNIVERSITY OF HAWAII

UNIVERSITY OF TOKYO

UNIVERSITY OF UTAH

WASHINGTON STATE UNIVERSITY

UNIVERSITY OF WASHINGTON

Printed in Japan by International Academic Printing Co., Ltd., Tokyo, Japan 


\section{Pacific Journal of Mathematics \\ Vol. 89 , No. 1 \\ May, 1980}

David Bressoud, A note on gap-frequency partitions ................ 1

John David Brillhart, A double inversion formula ................. 7

Frank Richard Deutsch, Günther Nürnberger and Ivan Singer, Weak

Chebyshev subspaces and alternation .......................

Edward Richard Fadell, The relationship between Ljusternik-Schnirelman

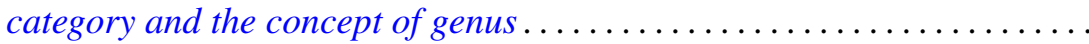

Harriet Jane Fell, On the zeros of convex combinations of polynomials.......

John Albert Fridy, An addendum to: "Tauberian theorems via block

dominated matrices" ..................................

Andrzej Granas, Ronald Bernard Guenther and John Walter Lee, Applications of topological transversality to differential equations. I. Some nonlinear

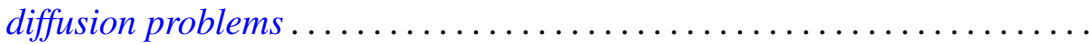

David E. Handelman and G. Renault, Actions of finite groups on self-injective

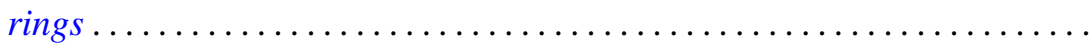

Michael Frank Hutchinson, Local $\Lambda$ sets for profinite groups .............

Arnold Samuel Kas, On the handlebody decomposition associated to a

Lefschetz fibration...

Hans Keller, On the lattice of all closed subspaces of a Hermitian space.....

P. S. Kenderov, Dense strong continuity of pointwise continuous

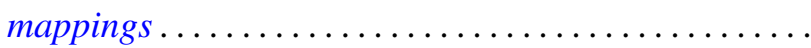

Robert Edward Kennedy, Krull rings.................

Jean Ann Larson, Richard Joseph Laver and George Frank McNulty,

Square-free and cube-free colorings of the ordinals ...

Viktor Losert and Harald Rindler, Cyclic vectors for $L^{p}(G)$

John Rowlay Martin and Edward D. Tymchatyn, Fixed point sets of

1-dimensional Peano continua...

Augusto Nobile, On equisingular families of isolated singularities ...

Kenneth Joseph Prevot, Imbedding smooth involutions in trivial bundles ...

Thomas Munro Price, Spanning surfaces for projective planes in four space.

Dave Riffelmacher, Sweedler's two-cocycles and Hochschild cohomology....

Niels Schwartz, Archimedean lattice-ordered fields that are algebraic over

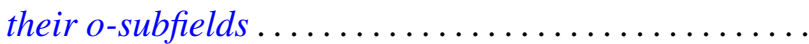

Chao-Liang Shen, A note on the automorphism groups of simple dimension groups.

Kenneth Barry Stolarsky, Mapping properties, growth, and uniqueness of

Vieta (infinite cosine) products ...

Warren James Wong, Maps on simple algebras preserving zero products. I.

The associative case ............................. 